\title{
Eftectiveness of Using Endothelial Colony-Forming Cells for Creating Tissue-Engineered Vascular Grafts under in vitro Conditions
}

DOI: 10.17691/stm2019.11.4.05

Received June 10, 2019

E.A. Velikanova, PhD, Researcher, Laboratory of Cell Technologies;

V.G. Matveeva, PhD, Senior Researcher, Laboratory of Cell Technologies;

E.O. Krivkina, Junior Researcher, Laboratory of Cell Technologies;

V.V. Sevostianova, PhD, Researcher, Laboratory of Cell Technologies;

M.Yu. Khanova, Junior Researcher, Laboratory of Cell Technologies;

Yu.A. Kudryavtseva, DSc, Head of Experimental and Clinical Cardiology Department;

L.V. Antonova, MD, DSc, Head of the Laboratory of Cell Technologies

Research Institute for Complex Issues of Cardiovascular Diseases, 6 Sosnovy Blvd, Kemerovo, 650002, Russia

The aim of the investigation was to assess the effectiveness of using endothelial colony-forming cells (ECFCs) for populating polymer vascular grafts under the conditions of a pulsatile bioreactor.

Materials and Methods. Grafts were fabricated by electrospinning from mixture of poly(3-hydroxybutyrate-co-3-hydroxyvalerate),

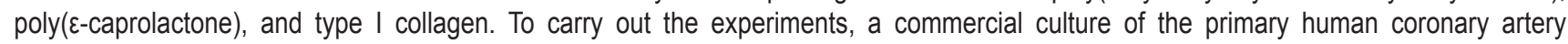
endothelial cells (HCAEC), a culture of the human umbilical vein endothelial cells (HUVEC), and ECFCs obtained from the peripheral blood of patients with IHD have been used. A suspension of the cells at a concentration of 700,000 per ml was introduced into the grafts, cultivated for 2 days under static conditions, then in the pulsatile reactor at shear stress of 1.27 dyn/ $/ \mathrm{cm}^{2}$ for 1 day, and at 2.85 dyn/ $/ \mathrm{cm}^{2}$ for another 5 days. The graft luminal surface underwent immunofluorescent staining for detecting CD31, VEGFR-2, CD144, vWF, F-actin markers and type IV collagen, after that, cell density was also calculated on $1 \mathrm{~mm}^{2}$ of the graft surface.

Results. Changes signifying their adaptation to shear stress have been registered in all cell cultures during culturing under dynamic conditions. They manifested themselves in intensified expression of the specific markers (VEGFR-2), increased secretory activity with regard to VWF and type IV collagen, ordered F-actin filaments and orientation of the cells along the flow, stimulation of intercellular junction formation. However, inevitably, the cells were partially washed off from the graft surface. The analysis of cellular layer density after dynamic cultivation allowed us to conclude that ECFCs had the strongest adhesion to the polymer and resistance to washing away with a flow.

Conclusion. ECFCs may be recognized to be the most promising culture for populating tissue-engineered vascular grafts owing to the possibility of being isolated from a readily available source (peripheral blood) and demonstrated good adaptation to the conditions of dynamic cultivation. However, significant reduction of cellular layer density caused by shear stress speaks of insufficient adhesion of the cells to the surface and requires further investigations to optimize a feeder layer covering the surface of a polymer scaffold.

Key words: tissue engineering; polymer vascular grafts; endothelial cells; pulsatile flow bioreactor.

\section{Introduction}

One of the aims of tissue engineering is to design a method for the reconstruction of the native vessel structure and to replace the damaged vessels with the obtained constructs [1]. The vessel wall is known to consist of the three functionally different layers. And a minimal requirement to the tissue-engineered vessel is formation of the internal endothelial layer which is expected to prevent graft thrombosing, a complication hindering the introduction of small-diameter tissueengineered vessels into clinical practice $[2,3]$.

Corresponding author: Elena A. Velikanova, e-mail: velikanova_ea@mail.ru 
There are different approaches to the implementation of this task including implantation of the grafts into the bloodstream which are modified to attract endothelial cells (ECs) for growing a vessel in situ; different variants of graft implantation into the tissue (for example, under the skin) to grow a vessel in vivo, and preliminary cultivation in vitro till an adequate endothelial layer is formed on the graft surface ready for implantation into bloodstream [1, 4].

The advantage of the latter approach relative to the method of growing in situ is that the functional endothelial layer created on the graft surface is capable of performing anti-thrombogenic function directly since the time of its implantation $[5,6]$.

However, this approach places demands on the process of preliminary cell culturing on a graft. It is necessary to provide favorable physiological conditions to the possible extent for cell culturing using special flow bioreactors for further successful adaptation to the body conditions and preservation of the endothelial layer after the implantation. Selection of optimal biochemical and mechanical effects on the cells in the period of endothelial layer formation in vitro is one of the main tasks which is to be solved in the process of fabricating these grafts $[7,8]$.

Another equally important task is a selection of the optimal cell source for luminal surface population. Researchers have used a wide spectrum of cells of different origin. Mature autologous ECs from various sources and ECs from the human umbilical vein have been widely employed; endothelial progenitor cells which can be harvested from the peripheral and cord blood, bone marrow, resident stem cells of the tissues also attract attention of the investigators $[9,10]$.

One of the most promising candidates for using in tissue engineering is endothelial colony-forming cells (ECFCs) which can be isolated from patient's peripheral blood and which combine the merits of different types of endothelial cells. The benefits of this type of cells are small invasiveness of their harvesting procedure and sufficient proliferating activity for creating an endothelial monolayer on the vascular graft surface under in vitro conditions. However, concern is caused by possible reduction of viability and proliferative activity in the obtained ECFCs in the culture due to advanced age of the patients in need of tissue-engineered vascular grafts or possible endothelial dysfunction [10].

The aim of our study was to assess the capabilities of using ECFCs to populate vascular grafts under pulsatile flow conditions by comparing their characteristics with other types of endothelial cells.

\section{Materials and Methods}

Fabrication of vascular grafts. Grafts were fabricated by electrospinning using Nanon-01A (MECC Co., Ltd., Japan) from poly(3-hydroxybutyrate-co-3hydroxyvalerate) (Sigma-Aldrich, USA) and $\operatorname{poly}(\varepsilon-$ caprolactone) (Sigma-Aldrich) in the ratio of $1: 2$ dissolved in 1,1,1,3,3,3-hexafluoro-2-propanol (SigmaAldrich). Type I collagen (Gibco, USA) was introduced into the inner third of the graft wall by feeding separately mixture of poly(3-hydroxybutyrate-co-3-hydroxyvalerate) with $\operatorname{poly}(\varepsilon$-caprolactone) and a solution of collagen in 1,1,1,3,3,3-hexafluoro-2-propanol in two separate syringes with a $5 \mathrm{mg} / \mathrm{ml}$ final concentration. The diameter of the winding collector was $4 \mathrm{~mm}$. A $27 \mathrm{G}$ needle was used to manufacture the inner third of the grafts, and a $22 \mathrm{G}$ needle was employed for other two thirds. Voltage on the needle amounted to $23 \mathrm{kV}$, the speed of polymer solution feeding was $0.3 \mathrm{ml} / \mathrm{h}$, collector rotation speed $200 \mathrm{rpm}$, needle-tip-to-collector distance $-15 \mathrm{~cm}$.

Cell cultivation. This investigation was approved by the Ethical Committee of the Research Institute for Complex Issues of Cardiovascular Diseases (Kemerovo). Written informed consent to use the biological material for the scientific study was obtained from all patients.

A commercial culture of primary human coronary artery endothelial cells (HCAEC) (Cell Applications, USA), a culture of human umbilical vein endothelial cells (HUVEC), and endothelial colony-forming cells (ECFCs) obtained from peripheral blood of patients with IHD have been used for the experiments.

According to the manufacturer's information, the primary human coronary artery endothelial cells were collected from the arteries of healthy donors using cryoconservation at the second passage (500,000 cells in the MesoEndo cell basal medium (Cell Applications), containing $10 \%$ fetal bovine serum and $10 \%$ dimethyl sulfoxide). The cells were defrosted and cultivated in compliance with the manufacturer's guidelines in the MesoEndo cell growth medium (Cell Applications).

Endothelial cells from human umbilical vein were isolated in accordance with the protocol of Jaffe et al. [11], umbilical cords were collected from women with uncomplicated pregnancy after successful delivery. The cells were cultivated in the MCDB 131 medium (Gibco) with the addition of the microvascular growth supplement (Gibco).

Endothelial colony-forming cells were obtained according to the protocol offered by Kolbe et al. [12]. A mononuclear fraction of peripheral blood from patients with IHD was isolated using a density gradient medium Histopaque 1077 (Sigma-Aldrich).

The obtained cell suspension was resuspended in the EGM-2MV growth medium (Lonza, Sweden), populated into $25 \mathrm{~cm}^{2}$ culture flasks coated with bovine type I collagen (Thermo Fisher, USA), and cultivated for a week under the conditions of a $\mathrm{CO}_{2}$-incubator. The cells were then detached from the flask surface with the Accutase solution (Sigma-Aldrich), reseeded, and cultivated on the plates covered with human fibronectin (SigmaAldrich). Further culture reseeding was performed when confluency reached $70-80 \%$.

Cells of the $6-8^{\text {th }}$ passages were used to populate the 
grafts. All experiments with cells were carried out under sterile conditions and cultivated in the $\mathrm{CO}_{2}$-incubator at $37^{\circ} \mathrm{C}, 5 \% \mathrm{CO}^{2}$.

Preparation of the grafts and population in bioreactors. The polymer grafts were sterilized by soaking in $70 \%$ ethyl alcohol for $60 \mathrm{~min}$. Then, in order to improve adhesive properties of the inner surface, they were placed for $12 \mathrm{~h}$ in the $10 \mu \mathrm{g} / \mathrm{ml}$ solution of human fibronectin (Sigma-Aldrich).

Cell suspension at a concentration of 700,000 per $\mathrm{ml}$ was introduced in the prepared grafts. In the first $8 \mathrm{~h}$ after the population, the grafts were turned over every $30 \mathrm{~min}$ to provide uniform population of the cells over the surface. In a day, the culture medium was changed. The grafts were cultured under static conditions for 2 days. Thereafter the grafts were connected to the system of pulsatile bioreactor (Figure 1) and cultured at preliminary settings providing shear stress of $1.27 \mathrm{dyn} / \mathrm{cm}^{2}$ for $24 \mathrm{~h}$. Then the stress was gradually increased to the values of the operating mode $-2.85 \mathrm{dyn} / \mathrm{cm}^{2}$. In this mode, the grafts were cultivated for another 5 days.

We continued to cultivate part of the grafts composing a control group under static conditions during the same period of time with the culture medium changed twice a day.

Analysis. After the experiment, the graft luminal surface underwent immunofluorescent staining. For this purpose, it was fixed in $4 \%$ formaldehyde solution for $10 \mathrm{~min}$, cut lengthwise, and fixed in the open position on the slides with their luminal surface turned upward. The cells on the luminal surface were stained for the markers of the endothelial cells: CD31, VEGFR-2, CD144, VWF, F-actin adhesion marker, and type IV collagen. Before staining for intracellular markers (vWF, F-actin) they were permeabilized with $0.1 \%$ solution of Triton X-100 (Sigma-Aldrich). F-actin marker was detected by staining with phalloidin conjugated to a fluorescent Alexa Fluor 568 dye (Thermo Fisher) in compliance with the manufacturer's instruction. Primary antibodies were used for the rest of the markers: mouse anti-CD31 (ab119339), rabbit anti-VEGFR-2 (ab2349), rabbit antiCD144 (ab33168), sheep anti-vWF — FITC (ab8822),

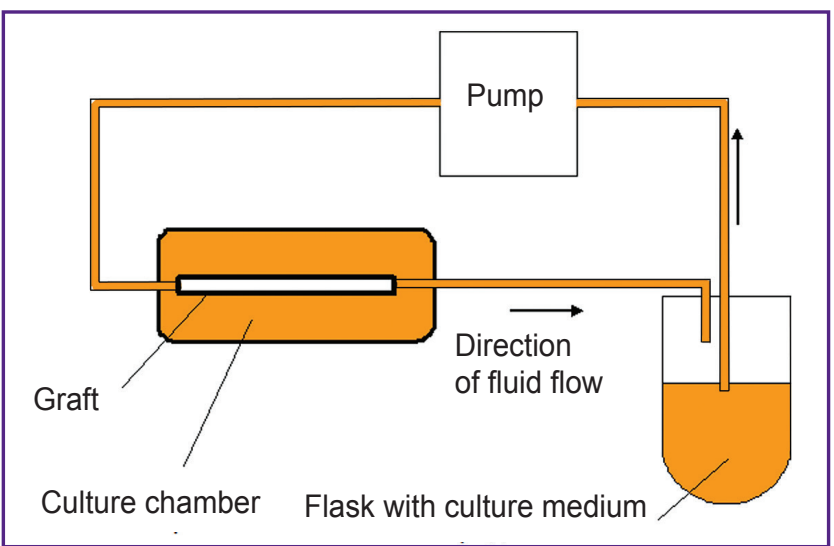

Figure 1. Schematic diagram of the flow bioreactor rabbit anti-collagen IV (ab8866) (Abcam, Great Britain) and secondary antibodies: donkey anti-mouse IgG highly cross-adsorbed - AF555 (A-31570), donkey antirabbit IgG highly cross-adsorbed - AF488 (A-21206) (Thermo Fisher). Incubation with primary antibodies was performed at $4^{\circ} \mathrm{C}$ for $16 \mathrm{~h}$, with the secondary antibodies at room temperature for $1 \mathrm{~h}$. $1 \%$ bovine serum albumin solution was used to block nonspecific binding. Specimens were counterstained with DAPI (SigmaAldrich). The ready slides were placed in ProLong reagent (Life Technologies, USA) under the glass. Preparations were analyzed using a laser scanning LSM 700 microscope (Carl Zeiss, Germany).

Additionally, population density on the graft surface was analyzed. A number of nuclei per field of view were counted on the DAPI-stained specimens, no less than 20 randomly selected fields of view were analyzed using $20 \times$ magnification. The results obtained were recounted per unit area and presented as unit $/ \mathrm{mm}^{2}$.

Data processing. The results obtained were statistically processed using Statistica 6.0 program. Compliance of data with normal distribution was assessed using Kolmagorov-Smirnov test. Data were presented as the median and quartiles (Me [25\%; 75\%]). Statistical significance of differences was evaluated using Mann-Whitney U-test with Bonferroni correction for multiple comparisons. Differences were considered significant at $p<0.05$.

\section{Results}

Evaluation of cell density on the graft surface. The microscopic analysis showed different tendency in populating the graft surface with the cells of various types. For the HCAEC culture it was characteristic to form local cell aggregates with tight intercellular junctions. The ECFCs were uniformly populated over the graft surface. The HUVEC culture demonstrated the most optimal population providing sufficiently full coverage of the surface with evident junctions between the cells.

Moderate population of the graft surface (161.35 [110.93; 252.11]) was typical for the ECFC culture when it was cultivated for 7 days under static conditions (Figure 2). At the same time, when it was cultivated for the same period of time under dynamic load this parameter turned out to be statistically significantly higher $(p<0.05)$ and amounted to 252.11 [201.69; 312.62].

The cells of the commercial HCAEC line also demonstrated moderate population of the graft surface and no difference was found between the cultivation under static or dynamic conditions: 211.77 [161.35; 262.20] and 201.69 [141.18; 211.77], respectively. However, population density for HCAEC in dynamics was significantly lower $(p<0.05)$ than for ECFCs under similar conditions (see Figure 2).

The greatest density of the surface coverage was obtained with the HUVEC culture under static conditions $-453.80 \quad[383.21 ; 594.99] \quad(p<0.05$ in 


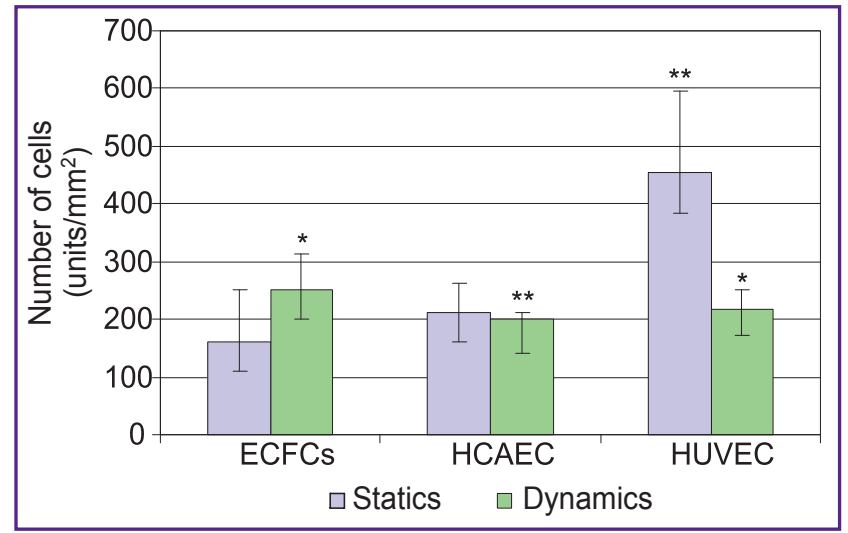

Figure 2. Number of cells on the graft luminal surface per unit area

* Statistically significant differences from the grafts cultured in statics, $p<0.05$; ${ }^{*}$ from the grafts populated with ECFCs, $p<0.05$

comparison with ECFCs and HCAEC). But the obtained endothelial monolayer was not sufficiently resistant to shear stress: during cultivation in dynamics the cell density was equal to $216.82[171.44 ; 252.11](p<0.05$ relative to statics) being comparable with the data obtained for ECFCs and HCAEC.

Immunofluorescence. On the basis of the results of staining with specific antibodies, the effect of the pulsatile flow on the cell phenotype, intercellular junctions, functional activity, and adhesion of the cells to the surface has been analyzed. It was found that a high expression level of the markers specific for endothelial cells CD31, VEGFR-2, CD144 was characteristic for all types of the cells used in the experiment. The cell phenotype remained the same during cultivation under static or pulsatile flow conditions.

The ECFC culture very intensively expressed the markers of the endothelial cells CD31, CD144 both under static and dynamic conditions of cultivation (Figure 3). The cells synthetized VWF and type IV collagen. In statics, there was noted a moderate expression of VEGFR-2 which increased substantially under shear stress. In statics and dynamics, marked F-actin filaments were detected in the cells though their greater orderliness and a tendency to orientation along the flow direction were noted in case of cultivation under dynamic conditions.

The HUVEC culture has also demonstrated high expression of endothelial markers (CD31, CD144) in statics and dynamics (Figure 4). It showed the highest intensity of intercellular junction formation (according to the stain on CD144) among all examined cultures. Substantial increase of VWF synthesis was noted under dynamic culturing conditions. In statics, the cells were characterized by low VEGFR-2 expression which intensified significantly in dynamics. A very intensive synthesis of collagen IV and F-actin assembly in statics and dynamics were observed.

The tendency described above persisted in relation to the expression of the specific markers for the HCAEC culture (Figure 5). They featured a weak prominence of intercellular junctions due to low density of the cell layer. Moderate expression of VEGFR-2 was noted both in statics and dynamics. The pulsatile flow significantly intensified synthesis of collagen IV by the cells. During cultivation under static conditions, ordered filaments of F-actin were found, whereas under pulsatile flow conditions the cells became much less spread, the filament structure disarranged.

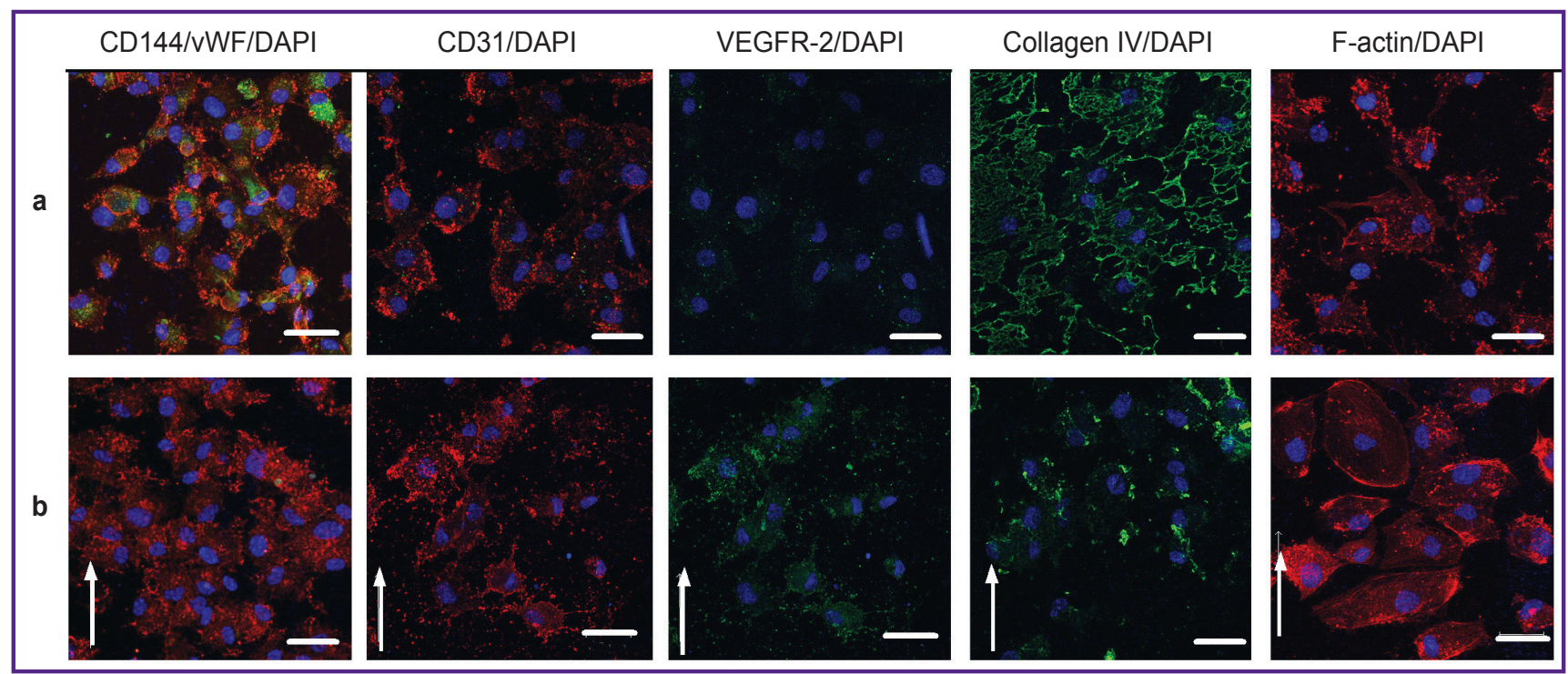

Figure 3. Immunofluorescent staining of the graft luminal surface populated with ECFCs:

(a) specimens cultured in statics; (b) specimens cultured under pulsatile flow conditions. Arrows indicate flow direction; $\times 630$; bar $-50 \mu \mathrm{m}$ 


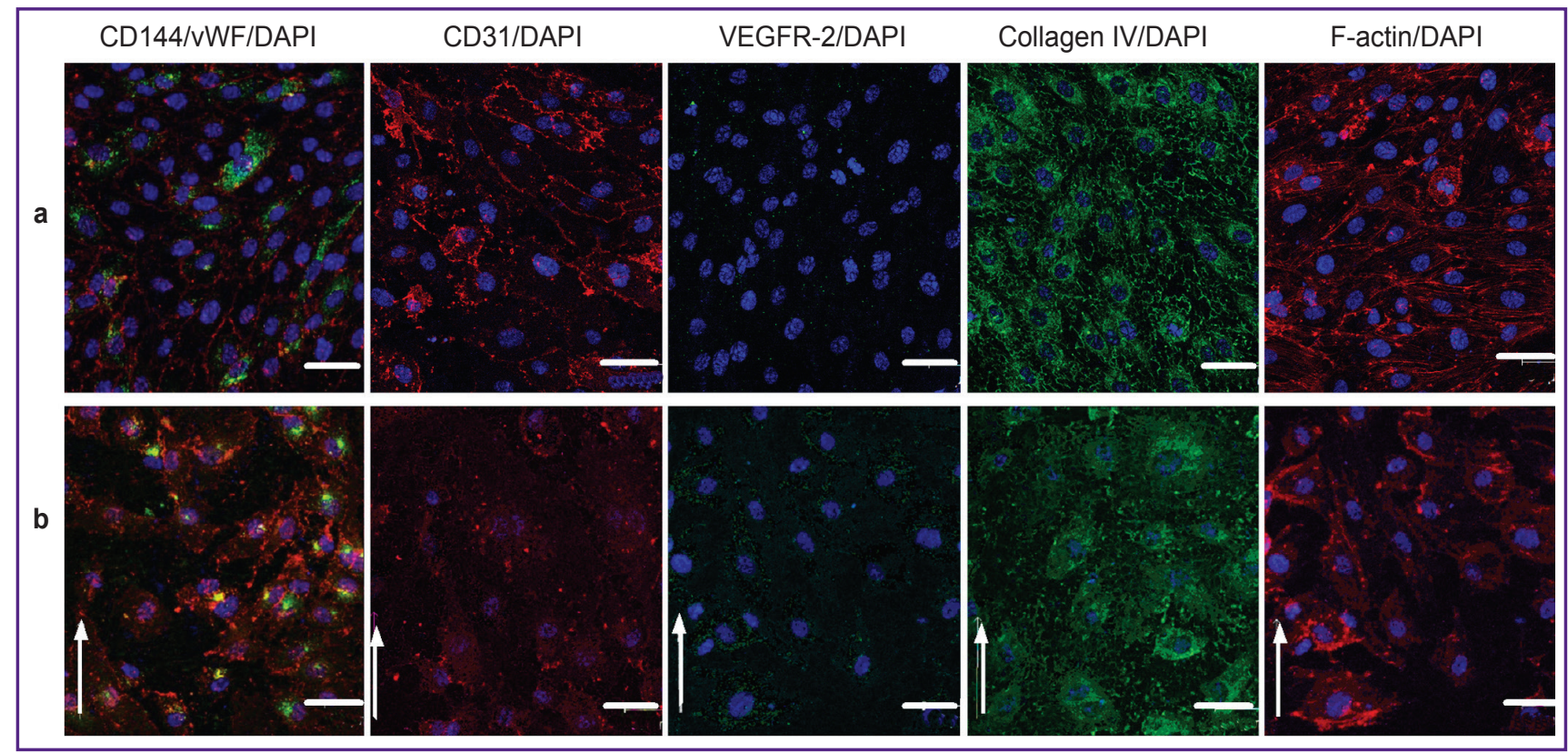

Figure 4. Immunofluorescent staining of the graft luminal surface populated with HUVEC:

(a) specimens cultured in statics; (b) specimens cultured under pulsatile flow conditions. Arrows indicate flow direction; $\times 630$; bar $-50 \mu \mathrm{m}$

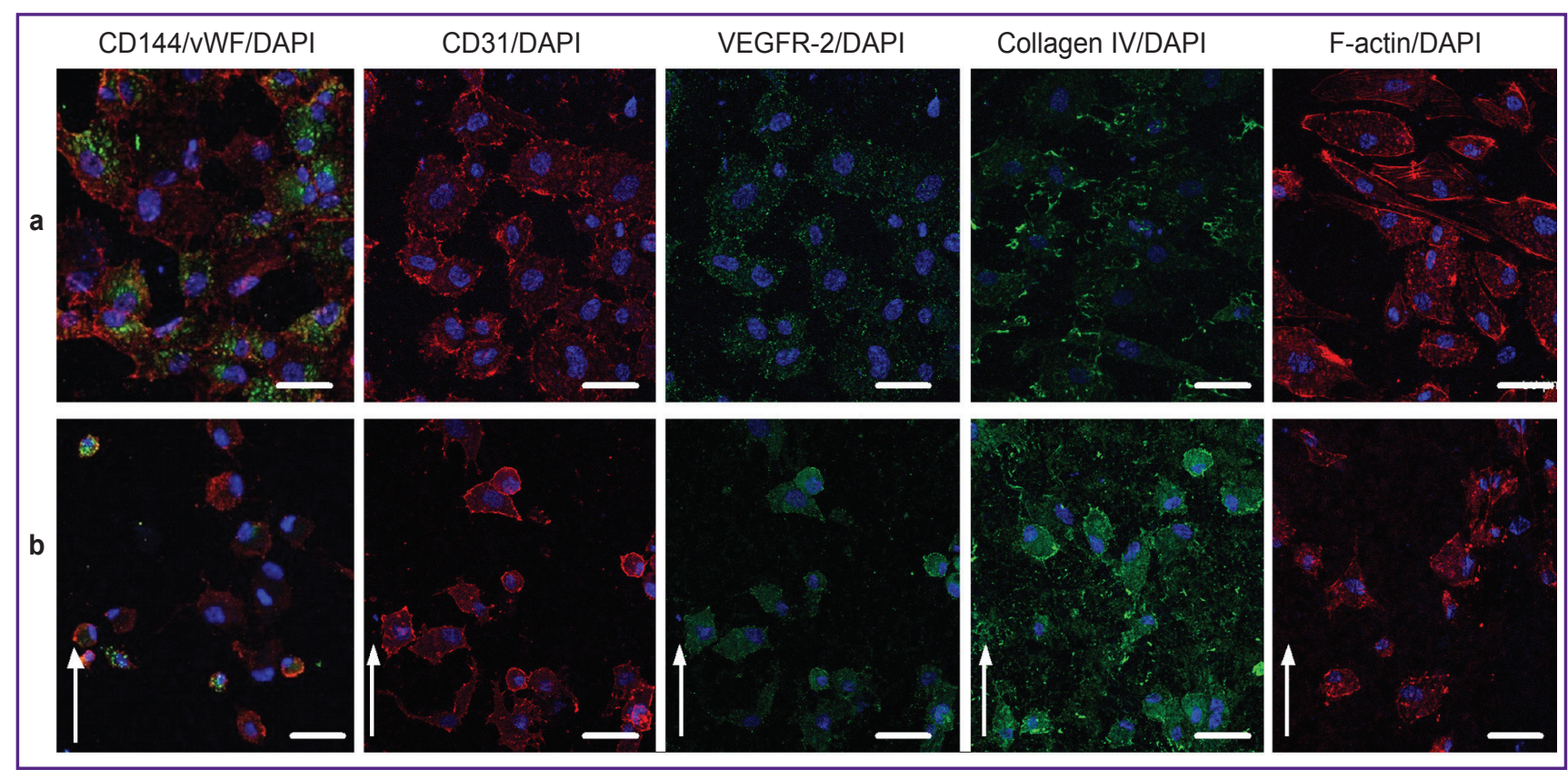

Figure 5. Immunofluorescent staining of the graft luminal surface populated with HCAEC:

(a) specimens cultured in statics; (b) specimens cultured under pulsatile flow conditions. Arrows indicate flow direction; $\times 630$; bar $-50 \mu \mathrm{m}$

\section{Discussion}

Shear stress which affects endothelial cells lining a vascular wall plays a central role in regulation of their vital activity. The action of this factor stimulates cell orientation relative to each other and flow direction, promotes intercellular junction formation, regulates activation of mechanosensors, intracellular signaling pathways, specific transcription factors, and expression of genes and proteins $[13,14]$. Therefore, to take into account this condition of cultivation is a key moment in creating cellpopulated tissue-engineered vascular grafts in vitro.

Endothelial cells in vivo are exposed to shear stress in the range of $5-20 \mathrm{dyn} / \mathrm{cm}^{2}$ [15]. And the investigators 
use different values of this parameter to simulate the conditions for culturing tissue-engineered vascular grafts. It has been shown that it is necessary to increase gradually the level of shear stress for successful adaptation of the populated endothelial cells to the flow forces [16]. Good endothelization of the grafts in vitro at low values of shear stress has also been noted $[17,18]$. Therefore, we used two different modes of operation in our work: adaptational, implying the exposure to shear stress of $1.27 \mathrm{dyn} / \mathrm{cm}^{2}$ for a day, and operating, with $2.85 \mathrm{dyn} / \mathrm{cm}^{2}$ shear stress and cultivation for 5 days. These cultivation modes were chosen in order to adapt the cells to the flow and to provide better cell retention on the surface and these two modes meet these requirements.

The analysis of cell population density under static and dynamic conditions showed that the best adaptation to dynamic conditions was characteristic for the ECFCs. After cultivation under static conditions for a week, the grafts populated with the ECFCs demonstrated relatively low density of the cell layer comparable with that for HCAEC and 2.8 times lower than for HUVEC. However, this value was 1.5 times greater $(p<0.05)$ on the grafts with ECFCs cultivated under pulsatile flow conditions than under static cultivation. Density of the cell layer on the HCAEC grafts after dynamic cultivation remained actually at the same level with statics (but significantly lower than on the grafts with ECFCs) whereas on the HUVEC grafts it decreased to the same level as on the grafts with ECFCs.

The obtained results showing the effect of the pulsatile flow on the cell physiology comply in whole with the data published in the literature [14]. The cultures of all selected cell lines (ECFC, HUVEC, HCAEC) have demonstrated the increase of synthetic activity and mechanotransduction during cultivation under dynamic conditions.

All cultures irrespective of the cultivation conditions maintained intensive expression of the endothelial cell marker CD31. For mature endothelial cells of the HCAEC culture, VEGFR-2 expression was characteristic both in statics and dynamics. The cells of ECFCs and HUVEC lines demonstrated low expression of this marker during static cultivation, but shear stress stimulated intensification of VEGFR-2 expression which is considered by some investigators as part of the mechanism promoting maintenance of endothelial cell viability in a blood vessel [19].

Active synthesis of vWF signifies functional activity of the endothelial cells. In our study, all types of the cells enhanced VWF synthesis under dynamic conditions relative to static conditions being a standard response of endothelial cells to shear stress [20].

It has been noted that fluid flow increases synthesis of type IV collagen which is a component of an extracellular matrix and vessel basal membrane. Its accumulation seems a necessary process for forming a stable endothelial layer.
Thus, changes signifying adaptation to fluid shear stress were registered in all cell cultures during their cultivation under dynamic conditions. But, inevitably, the cells were washed partially away from the graft surface. The data on cell layer density after dynamic cultivation give grounds to state that ECFCs display the best adhesion to the polymer and resistance to washing away by the flow. On the contrary, using HCAECs we failed to obtain an adequate layer even during static cultivation due to their relatively low proliferative potential and worse adhesion to the polymer. Considering the fact that collection of autologous mature endothelial cells is associated with high invasiveness and difficulties and that proliferative activity is not high in this type of cells, this culture may be recognized unsuitable for application in tissue engineering. The HUVEC culture showed good results in populating the grafts under static conditions, however, the obtained endothelial layer turned out to be not resistant enough to the flow forces. High proliferative capacity enabling a great bulk of cellular mass to be obtained is considered one of the HUVEC advantages. But this culture does not imply the possibility of allogenic application. In contrast, autologous ECFCs can be isolated from patient's blood and possess a sufficient proliferative potential for creation of personified tissueengineered vascular grafts [21].

\section{Conclusion}

Availability of the source (peripheral blood) and good adaptation to dynamic cultivation allow ECFCs to be recognized as the most promising culture for tissueengineered vascular graft population. However, significant reduction of cellular layer density caused by flow shear stress speaks of insufficient adhesion of the cells to the surface and requires further investigations to optimize a feeder layer covering the surface of a polymer scaffold.

Study funding. The work was supported by the Russian Scientific Foundation (grant No.17-75-20004 "Development of personalized tissue-engineered, smalldiameter vascular graft in vitro under pulsatile flow conditions").

Conflicts of interest. The authors declare no conflicts of interest related to this study.

\section{References}

1. Benrashid E., McCoy C.C., Youngwirth L.M., Kim J., Manson R.J., Otto J.C., Lawson J.H. Tissue engineered vascular grafts: origins, development, and current strategies for clinical application. Methods 2016; 99: 13-19, https://doi. org/10.1016/j.ymeth.2015.07.014.

2. Drews J.D., Miyachi H., Shinoka T. Tissue-engineered vascular grafts for congenital cardiac disease: clinical experience and current status. Trends Cardiovasc Med 2017; 27(8): 521-531, https://doi.org/10.1016/j.tcm.2017.06.013.

3. Melchiorri A.J., Hibino N., Fisher J.P. Strategies and techniques to enhance the in situ endothelialization of small- 
diameter biodegradable polymeric vascular grafts. Tissue Eng Part B Rev 2013; 19(4): 292-307, https://doi.org/10.1089/ten. teb.2012.0577.

4. Hoch E., Tovar G.E., Borchers K. Bioprinting of artificial blood vessels: current approaches towards a demanding goal. Eur J Cardiothorac Surg 2014; 46(5): 767-778, https://doi. org/10.1093/ejcts/ezu242.

5. Wang X., Lin P., Yao Q., Chen C. Development of smalldiameter vascular grafts. World J Surg 2007; 31(4): 682-689, https://doi.org/10.1007/s00268-006-0731-z.

6. Hauser S., Jung F., Pietzsch J. Human endothelial cell models in biomaterial research. Trends Biotechnol 2017; 35(3): 265-277, https://doi.org/10.1016/j.tibtech.2016. 09.007.

7. Couet F., Meghezi S., Mantovani D. Fetal development, mechanobiology and optimal control processes can improve vascular tissue regeneration in bioreactors: an integrative review. Med Eng Phys 2012; 34(3): 269-278, https://doi. org/10.1016/j.medengphy.2011.10.009.

8. van Haaften E.E., Bouten C.V.C., Kurniawan N.A. Vascular mechanobiology: towards control of in situ regeneration. Cells 2017; 6(3): E19, https://doi.org/10.3390/ cells6030019.

9. Kirton J.P., Xu Q. Endothelial precursors in vascular repair. Microvasc Res 2010; 79(3): 193-199, https://doi. org/10.1016/j.mvr.2010.02.009.

10. Lee P.S., Poh K.K. Endothelial progenitor cells in cardiovascular diseases. World J Stem Cells 2014; 6(3): 355366, https://doi.org/10.4252/wjsc.v6.i3.355.

11. Jaffe E.A., Nachman R.L., Becker C.G., Minick C.R. Culture of human endothelial cells derived from umbilical veins. Identification by morphologic and immunologic criteria. J Clin Invest 1973; 52(11): 2745-2756, https://doi.org/10.1172/ jci107470.

12. Kolbe M., Dohle E., Katerla D., Kirkpatrick C.J., Fuchs S. Enrichment of outgrowth endothelial cells in high and low colony-forming cultures from peripheral blood progenitors. Tissue Eng Part C Methods 2010; 16(5): 877-886, https://doi. org/10.1089/ten.tec.2009.0492.

13. Tzima E., Irani-Tehrani M., Kiosses W.B., Dejana E.,
Schultz D.A., Engelhardt B., Cao G., DeLisser H., Schwartz M.A. A mechanosensory complex that mediates the endothelial cell response to fluid shear stress. Nature 2005; 437(7057): 426-431, https://doi.org/10.1038/nature03952.

14. Nayak L., Lin Z., Jain M.K. "Go with the flow": how Krüppel-like factor 2 regulates the vasoprotective effects of shear stress. Antioxid Redox Signal 2011; 15(5): 1449-1461, https://doi.org/10.1089/ars.2010.3647.

15. Fisher A.B., Chien S., Barakat A.I., Nerem R.M. Endothelial cellular response to altered shear stress. Am J Physiol Lung Cell Mol Physiol 2001; 281(3): L529-L533, https://doi.org/10.1152/ajplung.2001.281.3.1529.

16. Liu H., Gong X., Jing X., Ding X., Yao Y., Huang Y., Fan $Y$. Shear stress with appropriate time-step and amplification enhances endothelial cell retention on vascular grafts. J Tissue Eng Regen Med 2017; 11(11): 2965-2978, https://doi.org/10.1002/term.2196.

17. Tondreau M.Y., Laterreur V., Gauvin R., Vallières K., Bourget J.M., Lacroix D., Tremblay C., Germain L., Ruel J., Auger F.A. Mechanical properties of endothelialized fibroblastderived vascular scaffolds stimulated in a bioreactor. Acta Biomater 2015; 18: 176-185, https://doi.org/10.1016/j. actbio.2015.02.026.

18. Wang X., Cooper S. Adhesion of endothelial cells and endothelial progenitor cells on peptide-linked polymers in shear flow. Tissue Eng Part A 2013; 19(9-10): 1113-1121, https://doi. org/10.1089/ten.tea.2011.0653.

19. dela Paz N.G., Walshe T.E., Leach L.L., SaintGeniez M., D'Amore P.A. Role of shear-stress-induced VEGF expression in endothelial cell survival. J Cell Sci 2012; 125(4): 831-843, https://doi.org/10.1242/jcs.084301.

20. Gogia S., Neelamegham S. Role of fluid shear stress in regulating VWF structure, function and related blood disorders. Biorheology 2015; 52(5-6): 319-335, https://doi.org/10.3233/ bir-15061.

21. Matveeva V.G., Khanova M.Yu., Velikanova E.A., Antonova L.V., Sardin E.S., Krutitsky S.S., Barbarash O.L. Isolation and characteristics of colony-forming endothelial cells from peripheral blood in patients with ischemic heart disease. Tsitologiia 2018; 60(8): 598-608. 